\title{
Risk of Overdose-Related Death for People with a History of Incarceration
}

\author{
Wen Qi Gan ${ }^{1}$, Stuart A. Kinner ${ }^{2,3,4}$, Tonia L. Nicholls ${ }^{2,5,6}$, Chloé Xavier ${ }^{1}$, Karen Urbanoski ${ }^{7}$, \\ Leigh Greiner $^{8}$, Jane A. Buxton ${ }^{1,2}$, Ruth E. Martin ${ }^{2}$, Katherine E. McLeod ${ }^{2}$, Hasina Samji ${ }^{1,9}$, \\ Seonaid Nolan ${ }^{10,11}$, Louise Meilleur ${ }^{12}$, Roshni Desai ${ }^{12}$, Soha Sabeti ${ }^{12}$ \& Amanda K. Slaunwhite ${ }^{1,2}$
}

\begin{abstract}
Author affiliations and addresses:
British Columbia Centre for Disease Control, Vancouver, BC, Canada, ${ }^{1}$ School of Population and Public Health, University of British Columbia, Vancouver, BC, Canada, ${ }^{2}$ Melbourne School of Population and Global Health, University of Melbourne, Melbourne, Victoria, Australia, ${ }^{3}$ Centre for Adolescent Health, Murdoch Children's Research Institute, Melbourne, Victoria, Australia, ${ }^{4}$ British Columbia Mental Health and Substance Use Services, Coquitlam, BC, Canada, ${ }^{5}$ Department of Psychiatry, University of British Columbia, Vancouver, BC, Canada, ${ }^{6}$ Canadian Institute for Substance Use Research, University of Victoria, Victoria, BC, Canada, ${ }^{7}$ British Columbia Corrections, Ministry of Public Safety and Solicitor General, Victoria, BC, Canada ${ }^{8}$ Faculty of Health Sciences, Simon Fraser University, Burnaby, BC, Canada, ${ }^{9}$ British Columbia Centre on Substance Use, Vancouver, BC, Canada, ${ }^{10}$ Department of Medicine, University of British Columbia, Vancouver, BC, Canada ${ }^{11}$ and First Nations Health Authority; Vancouver, BC, Canada $^{12}$
\end{abstract}

Running head: Previous Incarceration and Overdose-Related Death.

Word Count (text): 3,460.

Declaration of interests: None.

\section{Correspondence to:}

Dr. Wen Qi Gan

British Columbia Centre for Disease Control

655 West 12th Avenue, Vancouver, BC V5Z 4R4, Canada

E-mail: wenqi.gan@bccdc.ca

This is the author manuscript accepted for publication and has undergone full peer review but has not been through the copyediting, typesetting, pagination and proofreading process, which may lead to differences between this version and the Version of Record. Please cite this article as doi: $10.1111 /$ add.15293 


\begin{abstract}
(300 words)
Background and aims: Reported associations between previous incarceration and the risk of overdose-related death are substantially heterogeneous and previous studies are limited by an inability to control for confounding factors in risk assessment. This study investigated the associations of overdose-related death with previous incarceration and the number or cumulative duration of previous incarcerations, and individual or neighbourhood characteristics that may potentially modify the associations.

Design and setting: A cohort study using a 20\% random sample of residents in British Columbia, Canada.

Participants: 765,690 persons aged 23 years or older at baseline of January 1, 2015. Mean age was 50 years; $49 \%$ were males.

Measurements: Previous incarcerations that occurred during the 5-year exposure period (January 2010 to December 2014) were identified using provincial incarceration records. Overdose-related deaths that occurred during the 3-year follow-up period (January 2015 to December 2017) were identified using linked administrative health data. Baseline individual and neighbourhood characteristics were assessed using the provincial health insurance data.

Findings: In the cohort, 5,743 persons had an incarceration history during the exposure period, and 634 persons died from drug overdose during the follow-up period. The mortality rate was 897 and 22 per 100,000 person-years for persons who did and did not have an incarceration history, respectively. After adjusting for baseline individual and neighbourhood characteristics (without any interaction term), persons who had an incarceration history were 4.04 times (95\% confidence interval 3.23-5.06) more likely to die from drug overdose compared with persons without an incarceration history. The association was stronger for females, persons without diagnoses of substance use disorder, and persons without dispensation of opioids for pain or benzodiazepines $(P<0.001$ for each interaction term). There was no discernible linear trend between the number or cumulative duration of previous incarcerations and the risk of overdoserelated death.
\end{abstract}

Conclusions: Previous incarceration appears to be a major risk factor for overdose-related death.

Key words: cohort studies, confounding factors, drug overdose, incarceration, opioid-related disorders, prisons, prisoners, substance-related disorders, mortality. 


\section{INTRODUCTION}

Epidemiologic studies have shown that people released from correctional facilities are at substantially increased risk of overdose-related death compared with the general population [110]. A 12-year cohort study in Ontario, Canada, found that formerly incarcerated people were 20 times more likely than the general population to die from drug overdose [1]. Similarly, a 4-year cohort study in Washington State, USA, found that formerly incarcerated people were 13 times more likely than the general population to die from drug overdose [2]. A meta-analysis showed that overdose-related death was 27 times more likely for formerly incarcerated people than the general population [11]. Although previous studies consistently demonstrate that the risk of overdose-related death is elevated for formerly incarcerated people compared with the general population, the effect estimates vary substantially across these studies [11, 12].

It is notable that previous studies have important limitations in relative risk assessment. These studies include only one group of formerly incarcerated people, and the follow-up starts on dates of release from incarceration. Because this study design is not able to include a parallel control group that do not have an incarceration history, the general population is used as the reference group, and standardized mortality ratios (SMRs) are calculated to assess relative risk of overdose-related death for formerly incarcerated people. This study design is able to track absolute risk of overdose-related death over time, but it is not able to accurately assess relative risk of overdose-related death, because the calculation of SMRs is based on comparisons of aggregated mortality rates between formerly incarcerated people and the general population. These studies are ecological in nature, and are prone to ecological bias [13]. Moreover, these studies are not able to properly control for potential confounding factors. These limitations may partly explain the substantial heterogeneity in effect estimates across the previous studies. To 
address these limitations, we designed a cohort study that included an exposed group (persons who were previously incarcerated) and a parallel control group (persons who were not incarcerated) in British Columbia, Canada.

British Columbia has experienced unprecedented increases in fatal and non-fatal drug overdose since 2014 [14]. In April 2016, a provincial public health emergency was declared in response to the overdose epidemic [15], and the BC Provincial Overdose Cohort has been established to inform intervention efforts [16]. Recently, a panel of experts reviewed 1,854 overdose-related deaths in the province, and found that approximately two thirds of the decedents had an incarceration history or were under community correction supervision at the time of death [17]. This finding suggests that previous incarceration may be an important contributor to overdose-related death in the province. British Columbia has been most severely affected by drug overdose in Canada [14], it is still not known how the drug overdose epidemic has affected formerly incarcerated people in the province. Therefore, using a large random sample of the general population in the province, we conducted this cohort study to investigate:

(1) whether people with an incarceration history were more likely to die from drug overdose than people without an incarceration history after controlling for potential confounding factors; (2) whether the number or cumulative duration of previous incarcerations was associated with the risk of overdose-related death; and (3) individual and neighbourhood characteristics that may potentially modify the association between previous incarceration and the risk of overdoserelated death.

\section{METHODS}

\section{Data source}


In British Columbia, the Medical Services Plan managed by the provincial government provides universal health insurance for residents of the province [18]. Because enrollment in health insurance is mandatory, almost all residents in the province have registered with the Medical Services Plan. In the health insurance system, each person has a unique personal health number. On the basis of a series of deterministic and probabilistic linkage algorithms, records from various administrative data sources were linked at the individual level using name, date of birth, sex, and health insurance number [16].

\section{Study sample}

On the basis of the provincial health insurance's client roster during 2015-2017, a 20\% sample was drawn using simple random sampling [16]. All persons in the random sample who were aged 18 years or older on January 1, 2010, and still alive at baseline of January 1, 2015 were included in this study. Because almost all residents are registered with the mandatory health insurance, our study sample was representative of the general population in the province.

\section{Study design}

This is a cohort study including a 5-year exposure period (January 1, 2010 to December 31, 2014), and a 3-year follow-up period (January 1, 2015 to December 31, 2017). Persons who were released from provincial correctional facilities during the exposure period were identified using provincial incarceration records. Overdose-related deaths during the follow-up period were identified using linked administrative health data. The risk of overdose-related death was assessed for persons who had an incarceration history compared with persons without an incarceration history. 


\section{Measures}

\section{Outcome variable}

The outcome of interest in this study was overdose-related death. We identified overdoserelated deaths that occurred during the follow-up period (January 1, 2015 to December 31, 2017) using data from the BC Coroners Service and the Vital Statistics Agency. The BC Coroners Service is a provincial agency responsible for investigating unnatural and unexpected deaths in the province [19]. The BC Coroners Service defined an overdose-related death as a death involving a controlled and illegal street $\operatorname{drug}(\mathrm{s})$ (e.g., heroin, cocaine, MDMA, methamphetamine, fentanyl), or a medication(s) that was not prescribed to the decedent. In addition, some overdose-related deaths were identified using mortality data from the Vital Statistics Agency in combination with the opioid overdose diagnoses from the linked administrative health data including emergency department visits, ambulance services, hospital admissions, and outpatient visits (please see Overdose Identification in the Supporting $\underline{\text { Information for detailed information). }}$

\section{Predictor variable}

The predictor variable in this study was previous incarceration history. We identified persons who had an incarceration history during the exposure period (January 1, 2010 to December 31, 2014) using provincial incarceration records provided by the Ministry of Public Safety and the Solicitor General [20]. The incarceration data included records of incarceration for people aged 18 years or older, who were held in custody while awaiting trial or serving a custodial sentence of less than two years in a single incarceration event in the province. Each record included dates of reception into and release from a provincial correctional facility. 
Persons who did and did not have an incarceration history during the exposure period were categorized as formerly incarcerated persons and not incarcerated persons, respectively. For formerly incarcerated persons, we further calculated the number and cumulative duration of incarcerations during the exposure period.

\section{Covariates}

In addition to age and sex, the following individual and neighbourhood characteristics were also included as covariates. Presence of substance use disorder (SUD) or mental illness was determined using hospitalization and outpatient records. During the 5-year exposure period, if a person had one hospitalization record or two outpatient records in one year with diagnosis of SUD or mental illness [21], the person was identified as having SUD or mental illness. The revised Elixhauser comorbidity index was used to measure comorbidities [22], and was calculated using 27 categories of diseases recorded in the hospital admission data during the exposure period. Based on the data distribution, the comorbidity index was divided into three groups: none, 1 , or $\geq 2$. Dispensations of opioids for pain or benzodiazepines during the exposure period were identified using the provincial pharmaceutical data. Neighbourhood material deprivation index (NMDI) was used to indicate individual and neighbourhood socioeconomic status (SES), with quintiles 1 and 5 indicating the lowest and highest level of neighbourhood material deprivation, respectively [23]. Five regional Health Authorities were used to represent the region of residence. The 2014 residential postal codes were used to assign NMDI and regional Health Authority data to each person (please see Covariates in the Supporting Information for detailed information). 


\section{Statistical Methods}

We compared baseline individual and neighbourhood characteristics between persons who did and did not have an incarceration history using chi-square tests. We examined the associations of overdose-related death with previous incarceration as well as individual and neighbourhood characteristics using Cox proportional hazards regression models. During the follow-up period, persons were censored at date of death from other causes, or the end of followup (December 31, 2017). Person-time was determined for each person from the baseline of January 1, 2015 to date of overdose-related death, date of death from other causes, or the end of follow-up. For persons who were incarcerated during the follow-up period, cumulative duration of incarcerations during the follow-up period was calculated and was excluded from person-time of follow-up. We used the Kaplan-Meier curves to examine the proportional hazards assumption, and hazard ratios (HRs) and 95\% confidence intervals (CIs) of overdose-related death were calculated for persons who had an incarceration history compared with persons without an incarceration history.

We examined linear trends of the risk of overdose-related death with the number and cumulative duration of previous incarcerations during the exposure period. According to the data distribution, the number of previous incarcerations was divided into four groups: 1, 2, 3-4, and $\geq 5$; and cumulative duration was divided into quartiles: $<0.3,0.3-1.8,1.9-6.7$, and $\geq 6.8$ months. To investigate how individual and neighbourhood characteristics may potentially modify the association between previous incarceration and the risk of overdose-related death, we first examined statistical significance for the interaction term between each characteristic and the variable of previous incarceration in the fully adjusted model. For those characteristics that have a significant interaction term $(P<0.05)$, we further performed stratified analysis. 
All statistical tests were 2-sided and were performed using SAS Enterprise Guide 7.1 and $\mathrm{R}$ statistical computing environment (version 3.5.2). The survival package (version 3.1-12) was used for the Cox regression analyses [24]. The analyses were not pre-registered, so the results should be considered exploratory.

\section{RESULTS}

This cohort study included 765,690 persons at baseline. During the 5-year exposure period, 5,743 persons (incarceration prevalence 750 per 100,000 population) had an incarceration history. Compared with persons without an incarceration history, persons who had an incarceration history were younger (mean age 39 vs. 50 years at baseline), and were more likely to be male ( $87 \%$ vs. $49 \%$ ), have SUD or SUD combined with mental illness, be dispensed opioids for pain or benzodiazepines, and live in more materially deprived neighborhoods or have missing residential address. There was no significant difference in the comorbidity index between the two groups (Table 1).

During the 3-year follow-up period, 634 persons died from drug overdose (overall mortality rate 28 per 100,000 person-years), including 515 deaths (81\%) were identified from the BC Coroners Service and additional 119 deaths (19\%) were identified only from the Vital Statistics Agency. There were 140 overdose-related deaths among persons with an incarceration history (mortality rate 897 per 100,000 person-years), and 494 overdose-related deaths among persons without an incarceration history (mortality rate 22 per 100,000 person-years). In total, 765,056 persons were censored due to deaths from other causes $(3 \%)$ or the end of follow-up (97\%). The Kaplan-Meier curves for the risk of overdose-related death by previous incarceration history and each covariate showed no violation of the proportional hazards assumption. Persons 
who had an incarceration history were 41.42 (95\% CI 34.33-9.98) times more likely to die from drug overdose compared with persons without an incarceration history (Table 2). After adjusting for individual and neighbourhood characteristics (without inclusion of any interaction term), the effect estimate was substantially attenuated, but remained significantly elevated (adjusted HR 4.04; 95\% CI 3.23-5.06) (Table 2). Among these characteristics, SUD was the most important confounder, adjusting for SUD reduced the unadjusted HR by $84 \%$. Whereas each of other characteristics including mental illness, sex, dispensation of opioids for pain, dispensation of benzodiazepines, age group, and NMDI reduced the unadjusted HR by $13 \%$ to $28 \%$. In the model including SUD, further adjusting for these six characteristics reduced the unadjusted HR by an additional 7\% (Table 3). Furthermore, unadjusted and adjusted analyses showed that the risk of overdose-related death was also significantly increased for younger age groups, males, persons with SUD or mental illness, persons dispensed opioids for pain or benzodiazepines, persons with the comorbidity index $\geq 2$, and persons living in more materially deprived neighbourhoods (Table 2).

When stratified by the number of incarcerations during the exposure period, there was a linear trend between the number of previous incarcerations and unadjusted HRs of overdoserelated death. However, after adjusting for the individual and neighbourhood characteristics, the linear trend was no longer evident (Figure 1). Similarly, when stratified by quartiles of cumulative duration of previous incarcerations during the exposure period, there was a linear trend between quartiles of cumulative duration and unadjusted HRs of overdose-related death. The linear trend was no longer evident after adjusting for the individual and neighbourhood characteristics (Figure 2). 
The stratified analyses by individual and neighbourhood characteristics showed that the association between previous incarceration and the risk of overdose-related death was stronger for females than males, for persons who did not have SUD (with or without mental illness) than persons who had SUD, and for persons who were not dispensed opioids for pain or benzodiazepines than persons who were dispensed these medications $(P<0.001$ for each of the interaction terms) (Figure 3, Table S1). The stratified analysis by quintiles of NMDI showed that the association was significantly heterogeneous $(P=0.032$ for the interaction term), with no consistent trend across quintiles of NMDI.

\section{DISCUSSION}

On the basis of a large random sample of the general population in British Columbia, we found that people who had an incarceration history during the 5-year exposure period were four times more likely to die from drug overdose during the 2-year follow-up period compared with people without an incarceration history during the exposure period. Moreover, we found that this relationship was stronger among females, persons who did not have previous diagnoses of SUD, and persons who were not dispensed opioids for pain or benzodiazepines. We did not find a discernible linear trend between the number or cumulative duration of previous incarcerations and the risk of overdose-related death.

Based on our detailed literature review, this is the first cohort study including both an exposed group and a parallel control group. Relative risk of overdose-related death was assessed by comparing individual persons who did and did not have an incarceration history, and adjusting for individual and neighbourhood characteristics. Our analysis shows that SUD is the most important confounder, adjusting for SUD reduced the unadjusted HR by $84 \%$, further 
adjusting for other covariates reduced the unadjusted HR by an additional 7\% (Table 3). These data suggest that relative risk assessment without adjustment for covariates, especially SUD, would substantially overestimate the true risk of overdose-related death. Previous studies assessed relative risk of overdose-related death by comparing mortality rates between formerly incarcerated people and the general population after controlling for age and sex, but not SUD, and thus might overestimate the risk of overdose-related death for formerly incarcerated people [1-10]. In addition, our cohort study only included overdose-related deaths that occurred during the follow-up period. The difference in study designs may also contribute to the discrepancy in effect estimates between our study and the previous studies. Compared with the previous studies, our study is more reflective of intermediate and long-term effects of previous incarceration on the risk of overdose-related death.

The previous studies reported widely varying effect estimates for the association between previous incarceration and the risk of overdose-related death [1-12]. A meta-analysis reported that overdose mortality rates ranged from 29 to 537 per 100,000 person-years among formerly incarcerated people [12], which are lower than our finding of 897 per 100,000 person-years, although our study design tended to yield smaller mortality rate due to excluding overdoserelated deaths that occurred during the exposure period. Another meta-analysis reported a pooled SMR of 27 (95\% CI 10-76) for formerly incarcerated people compared with the general population [11]. Our unadjusted HR of 41.42 is within the $95 \%$ CI, but our adjusted HR of 4.04 is much smaller than the pooled SMR, and most previously reported SMRs [1-4, 6-12].

Our stratified analysis showed that the association between previous incarceration and the risk of overdose-related death was stronger for females than males, which is consistent with the previous findings $[2-4,6]$. We also observed that the association was stronger for persons who 
did not have previous diagnoses of SUD, or previous dispensation of opioids for pain or benzodiazepines. It is possible that persons who did not have the previous records were more likely to be new drug users in the community during the follow-up period, thus they were less knowledgeable and less experienced in drug use, and less likely to have access to the harm reduction services in the community; whereas persons who had the previous records were more likely to receive opioid agonist therapy and take advantage of the harm reduction services in the community, which are proven effective in reducing the risk of overdose-related death [25-28]. In addition, the stratified analysis showed that the association between previous incarceration and the risk of overdose-related death was weaker for persons who had previous SUD than those who did not have previous SUD (HR 2.49 vs. 16.44, Figure 1 and Table S1), suggesting that decreased physiological tolerance to drugs after a period of abstinence in custody did not play a key role as an underlying pathway for the observed association in our study [29, 30].

Our analysis showed that quintiles of NMDI were associated with the risk of overdoserelated death in a dose-response manner, people who lived in more deprived neighbourhoods were more likely to die from drug overdose compared with those living in less deprived neighbourhoods. This relationship was more evident for persons who did not have residential address, which might indicate unstable housing or homelessness (Table 2). To effectively address the overdose epidemic, more attention should be devoted to materially deprived neighborhoods.

This study has some limitations that should be considered when interpreting the findings. First, this study was based on linked administrative health data. Some individual information such as SES was not available, and thus could not be controlled for in the analyses. We used NMDI to reflect individual and neighborhood SES, this approach was able to reduce the 
influence of individual SES on the effect estimates [31,32]. Second, incarceration information was only available for provincial facilities, but was not available for federal facilities, where sentences are two years or longer. This limitation would potentially result in exposure misclassification, leading to underestimation of the true risk of overdose-related death. Finally, identification of overdose-related deaths was based on linked administrative health data. It is possible that some intentional drug overdose deaths such as suicides may have been misclassified as accidental overdose-related deaths [33, 34], and some overdose-related deaths may have been misclassified as respiratory or cardiovascular causes. However, in cases of sudden and unexpected deaths that appear to be due to an accident, suicide, or homicide, the provincial Coroners Service routinely conducts investigations including autopsy [35]. In our study, $81 \%$ of overdose-related deaths were identified from the BC Coroners Service records, thus the misclassification of overdose-related deaths was not likely to be common.

\section{CONCLUSIONS}

This large cohort study has shown that the drug overdose epidemic in British Columbia more severely affected formerly incarcerated people than the general population. Drug overdose has been widely identified as the leading cause of death for people released from incarceration [1-4, 6-10]. Worldwide, more than 30 million people are released from incarceration every year [36], overdose-related death is a serious concern for these socially and economically marginalised individuals. Research is needed to better understand the mechanisms underlying the increased risk of overdose-related death among formerly incarcerated people [37].

Although the risk of overdose-related death is alarmingly high among formerly incarcerated people, opioid use disorder is treatable and drug overdose is preventable [30, 38]. 
According to the WHO recommendations for preventing overdose-related deaths [30], it is critical to provide social and economic support such as housing and employment for community reintegration, and provide specific interventions in the community including uninterrupted health care services and treatment for SUD and mental illness [39].

Cumulative evidence has demonstrated that incarceration is associated with substantial adverse health and socioeconomic impacts not only for incarcerated persons but also for their families and communities [40-42]. In particular, after release from incarceration, there is a lack of support from the justice system for community reintegration [43, 44]. Because of widespread discrimination, unstable housing, unemployment, and poor family and social support $[45,46]$, transitioning back into the community is extremely difficult, if not seemingly impossible [37]. Given the considerable negative impacts, it is important to avoid unnecessary and excessive use of incarceration [47]. Meanwhile, further research is needed to identify more effective alternatives to incarceration that will not severely disrupt family and social relationships.

Acknowledgements: Data for the analyses were provided by the BC Coroners Service, the BC Emergency Health Services, the BC Drug and Poison Information Centre, the BC Ministry of Health (Discharge Abstract Database, National Ambulatory Care Reporting System, Medical Services Plan, and PharmaNet), the Ministry of Public Safety and the Solicitor General, and the Emergency Departments in Interior, Island, and Northern Health Authorities. All inferences, opinions, and conclusions are those of the authors, and do not reflect the opinions or policies of the Data Stewards. 


\section{Figure Legends}

Figure 1. Hazard ratios of overdose-related death for persons with an incarceration history compared with those without an incarceration history, stratified by the number of previous incarcerations during the exposure period.

Adjusted HRs were controlled for all the characteristics in Table 1.

$\mathrm{HR}=$ hazard ratio.

Figure 2. Hazard ratios of overdose-related death for persons with an incarceration history compared with those without an incarceration history, stratified by quartiles of cumulative duration of previous incarcerations during the exposure period.

Adjusted HRs were controlled for all the characteristics in Table 1.

$\mathrm{HR}=$ hazard ratio

Figure 3. Adjusted hazard ratios of overdose-related death for persons with an incarceration history compared with those without an incarceration history, stratified by individual and neighborhood characteristics.

Hazard ratios were adjusted for the characteristics in Table 1 without including any interaction term. $P<0.001$ for the interaction term with sex, SUD and mental illness, dispensation of benzodiazepines, and dispensation of opioids for pain; $P=0.033$ for the interaction term with NMDI.

$\mathrm{NMDI}=$ neighbourhood material deprivation index; SUD = substance use disorder. 
Table 1. Baseline individual and neighborhood characteristics for persons who did and did not have an incarceration history during the exposure period. ${ }^{1}$

\begin{tabular}{|c|c|c|c|}
\hline Characteristic & $\begin{array}{l}\text { Persons without an } \\
\text { incarcerated history } \\
\quad(\mathrm{n}=759,947)\end{array}$ & $\begin{array}{l}\text { Persons with an } \\
\text { incarcerated history } \\
\quad(\mathrm{n}=5,743)\end{array}$ & $\begin{array}{c}\text { Total } \\
(\mathrm{n}=765,690)\end{array}$ \\
\hline \multicolumn{4}{|l|}{ Age group (years) } \\
\hline$<35$ & $175,778(23.1)$ & $2,325(40.5)$ & $178,103(23.3)$ \\
\hline $35-44$ & $132,069(17.4)$ & $1,657(28.9)$ & $133,726(17.5)$ \\
\hline $45-54$ & $147,090(19.4)$ & $1,259(21.9)$ & $148,349(19.4)$ \\
\hline$\geq 55$ & $305,010(40.1)$ & $502(8.7)$ & $305,512(39.9)$ \\
\hline \multicolumn{4}{|l|}{ Sex } \\
\hline Males & $371,258(48.9)$ & 4,990 (86.9) & $376,248(49.1)$ \\
\hline Females & $388,689(51.1)$ & $753(13.1)$ & $389,442(50.9)$ \\
\hline \multicolumn{4}{|l|}{ SUD and mental illness } \\
\hline No SUD or mental illness & $627,360(82.6)$ & $2,930(51.0)$ & $630,290(82.3)$ \\
\hline SUD only & $3,952(0.5)$ & $601(10.5)$ & $4,553(0.6)$ \\
\hline Mental illness only & $120,807(15.9)$ & $909(15.8)$ & $121,716(15.9)$ \\
\hline SUD and mental illness & $7,828(1.0)$ & $1,303(22.7)$ & $9,131(1.2)$ \\
\hline \multicolumn{4}{|l|}{ Elixhauser comorbidity index ${ }^{2}$} \\
\hline None & $675,498(88.9)$ & $5,131(89.3)$ & $680,629(88.9)$ \\
\hline 1 & $35,365(4.7)$ & $272(4.7)$ & $35,637(4.7)$ \\
\hline$\geq 2$ & $49,084(6.5)$ & $340(5.9)$ & $49,424(6.5)$ \\
\hline \multicolumn{4}{|l|}{ Dispensed benzodiazepines } \\
\hline No & $637,369(83.9)$ & $3,903(68.0)$ & $641,272(83.8)$ \\
\hline Yes & $122,578(16.1)$ & $1,840(32.0)$ & $124,418(16.2)$ \\
\hline \multicolumn{4}{|l|}{ Dispensed opioids for pain } \\
\hline No & $484,404(63.7)$ & 2,291 (39.9) & 486,695 (63.6) \\
\hline Yes & $275,543(36.3)$ & $3,452(60.1)$ & $278,995(36.4)$ \\
\hline \multicolumn{4}{|l|}{ NMDI (quintiles) } \\
\hline Q 1 (lowest) & $133,195(17.5)$ & $403(7.0)$ & $133,598(17.4)$ \\
\hline Q 2 & $123,098(16.2)$ & $540(9.4)$ & $123,638(16.1)$ \\
\hline Q 3 & $126,115(16.6)$ & $615(10.7)$ & $126,730(16.6)$ \\
\hline Q 4 & $129,299(17.0)$ & 897 (15.6) & $130,196(17.0)$ \\
\hline Q 5 (highest) & $130,338(17.2)$ & $1,410(24.6)$ & $131,748(17.2)$ \\
\hline Unknown & $117,902(15.5)$ & $1,878(32.7)$ & $119,780(15.6)$ \\
\hline
\end{tabular}

This article is protected by copyright. All rights reserved. 
Health Authority region of residence

Interior
Fraser
Vancouver Coastal
Island
Northern
Unknown

\begin{tabular}{ccc}
$115,033(15.1)$ & $790(13.8)$ & $115,823(15.1)$ \\
$247,692(32.6)$ & $1,852(32.2)$ & $249,544(32.6)$ \\
$188,918(24.9)$ & $1,025(17.8)$ & $189,943(24.8)$ \\
$118,994(15.7)$ & $840(14.6)$ & $119,834(15.7)$ \\
$43,060(5.7)$ & $517(9.0)$ & $43,577(5.7)$ \\
$46,250(6.1)$ & $719(12.5)$ & $46,969(6.1)$ \\
\hline
\end{tabular}

NMDI = neighbourhood material deprivation index; SUD = substance use disorder.

${ }^{1} P<0.01$ for the comparison of each characteristic between the two groups, unless otherwise specified. ${ }^{2} P=0.249$ for the comparison between the two groups. 
Table 2. Hazard ratios of overdose-related death in relation to individual and neighborhood characteristics. ${ }^{1}$

\begin{tabular}{|c|c|c|}
\hline Characteristic & $\begin{array}{l}\text { Unadjusted HR } \\
\quad(95 \% \mathrm{CI})\end{array}$ & $\begin{array}{l}\text { Adjusted HR } \\
(95 \% \mathrm{CI})^{2}\end{array}$ \\
\hline \multicolumn{3}{|l|}{ Age group (year) } \\
\hline$\geq 55$ & Reference (1.00) & Reference (1.00) \\
\hline$<35$ & $2.56(2.05-3.20)$ & $2.44(1.91-3.11)$ \\
\hline $35-44$ & $2.61(2.06-3.31)$ & $2.05(1.59-2.64)$ \\
\hline $45-54$ & $2.53(2.00-3.19)$ & $2.02(1.59-2.58)$ \\
\hline \multicolumn{3}{|l|}{ Sex } \\
\hline Females & Reference (1.00) & Reference (1.00) \\
\hline Males & $3.84(3.17-4.64)$ & $3.41(2.80-4.15)$ \\
\hline \multicolumn{3}{|l|}{ SUD and mental illness } \\
\hline No SUD or mental illness & Reference (1.00) & Reference (1.00) \\
\hline SUD only & $42.63(32.95-55.16)$ & $11.50(8.63-15.34)$ \\
\hline Mental illness only & $2.52(2.03-3.12)$ & $1.85(1.47-2.34)$ \\
\hline SUD and mental illness & $51.63(42.70-62.44)$ & $11.34(8.85-14.53)$ \\
\hline \multicolumn{3}{|l|}{ Elixhauser comorbidity index } \\
\hline None & Reference (1.00) & Reference (1.00) \\
\hline 1 & $1.52(1.10-2.10)$ & $0.98(0.70-1.37)$ \\
\hline$\geq 2$ & $2.64(2.10-3.31)$ & $1.63(1.28-2.09)$ \\
\hline \multicolumn{3}{|l|}{ Dispensed benzodiazepines } \\
\hline No & Reference (1.00) & Reference (1.00) \\
\hline Yes & $4.70(4.02-5.50)$ & $1.90(1.57-2.29)$ \\
\hline \multicolumn{3}{|l|}{ Dispensed opioids for pain } \\
\hline No & Reference (1.00) & Reference (1.00) \\
\hline Yes & $5.20(4.35-6.22)$ & $2.44(2.00-2.98)$ \\
\hline \multicolumn{3}{|l|}{ NMDI (quintiles) } \\
\hline Q 1 (lowest) & Reference (1.00) & Reference (1.00) \\
\hline Q 2 & $0.92(0.65-1.31)$ & $0.85(0.60-1.22)$ \\
\hline Q 3 & $1.30(0.95-1.80)$ & $1.19(0.86-1.65)$ \\
\hline Q 4 & $1.73(1.28-2.33)$ & $1.43(1.05-1.94)$ \\
\hline Q 5 (highest) & $2.35(1.77-3.13)$ & $1.59(1.19-2.14)$ \\
\hline Unknown & $2.56(1.92-3.41)$ & $2.11(1.57-2.84)$ \\
\hline
\end{tabular}

This article is protected by copyright. All rights reserved. 
Health Authority region of residence

\begin{tabular}{lcc} 
Northern & Reference $(1.00)$ & Reference $(1.00)$ \\
Interior & $1.28(0.87-1.88)$ & $1.43(0.97-2.10)$ \\
Fraser & $1.14(0.79-1.63)$ & $1.33(0.93-1.91)$ \\
Vancouver Coastal & $1.07(0.74-1.55)$ & $1.34(0.92-1.96)$ \\
Island & $0.99(0.67-1.46)$ & $1.15(0.77-1.70)$ \\
Unknown & $0.32(0.17-0.62)$ & $0.51(0.25-1.02)$ \\
Previous incarceration & & \\
No & Reference $(1.00)$ & Reference $(1.00)$ \\
Yes & $41.42(34.33-49.98)$ & $4.04(3.23-5.06)$ \\
\hline
\end{tabular}

$\mathrm{CI}=$ confidence interval HR = hazard ratio NMDI = neighbourhood material deprivation index; SUD = substance use disorder.

${ }^{1}$ Hazard ratios were calculated without inclusion of any interaction term.

${ }^{2}$ Adjusted for all the characteristics in Table 1. 
Table 3. Hazard ratios and relative decreases of overdose-related death for persons with an incarceration history compared with persons without an incarceration history after adjusting for a single or multiple characteristics.

\begin{tabular}{|c|c|c|c|c|c|c|}
\hline \multirow[b]{2}{*}{ Characteristics } & \multicolumn{3}{|c|}{ Single Adjustment ${ }^{1}$} & \multicolumn{3}{|c|}{ Multiple Adjustment $^{2}$} \\
\hline & HR & $95 \% \mathrm{CI}$ & $\begin{array}{c}\text { Decrease } \\
(\%)^{3}\end{array}$ & HR & $95 \% \mathrm{CI}$ & $\begin{array}{c}\text { Decrease } \\
(\%)^{3}\end{array}$ \\
\hline SUD & 6.84 & $5.46-8.56$ & -83.5 & 6.84 & $5.46-8.56$ & -83.5 \\
\hline Mental illness & 29.81 & $24.59-36.15$ & -28.0 & 6.70 & $5.35-8.39$ & -83.8 \\
\hline Sex & 29.99 & $24.75-36.35$ & -27.6 & 5.38 & $4.30-6.73$ & -87.0 \\
\hline Dispensed opioids for pain & 30.31 & $25.07-36.65$ & -26.8 & 5.04 & $4.03-6.29$ & -87.8 \\
\hline Dispensed benzodiazepines & 31.54 & $26.06-38.18$ & -23.9 & 4.95 & $3.97-6.19$ & -88.0 \\
\hline Age group & 34.95 & $28.83-42.37$ & -15.6 & 4.38 & $3.50-5.48$ & -89.4 \\
\hline NMDI (quintiles) & 36.22 & $29.89-43.89$ & -12.6 & 4.06 & $3.25-5.08$ & -90.2 \\
\hline Elixhauser comorbidity index & 41.55 & $34.43-50.13$ & 0.3 & 4.06 & $3.25-5.08$ & -90.2 \\
\hline Health Authority region & 43.84 & $36.31-52.94$ & 5.8 & $4.05^{4}$ & $3.23-5.07^{4}$ & -90.2 \\
\hline
\end{tabular}

$\mathrm{CI}=$ confidence interval $\mathrm{HR}=$ hazard ratio NMDI = neighborhood material deprivation index SUD = substance use disorder.

${ }^{1}$ HRs were adjusted for a single covariate in each row.

${ }^{2}$ HRs were cumulatively adjusted for multiple covariates (covariate in a row and covariates above the row. E.g., for the row of sex, HR was adjusted for sex as well as mental illness and SUD).

${ }^{3}$ Decrease $(\%)=[(\mathrm{HR}-41.42) / 41.42] \times 100$. Here, 41.42 is the unadjusted HR (see Table 2$)$.

${ }^{4}$ The HR (95\% CI) is slightly different from the HR in Table 2 (HR 4.04, 95\% CI 3.23-5.06) because SUD and mental illness are treated as two separate covariates here. 
Figure 1. Hazard ratios of overdose-related death for persons with an incarceration history compared with those without an incarceration history, stratified by the number of previous incarcerations during the exposure period.

Adjusted HRs were controlled for all the characteristics in Table 1.

$\mathrm{HR}=$ hazard ratio.

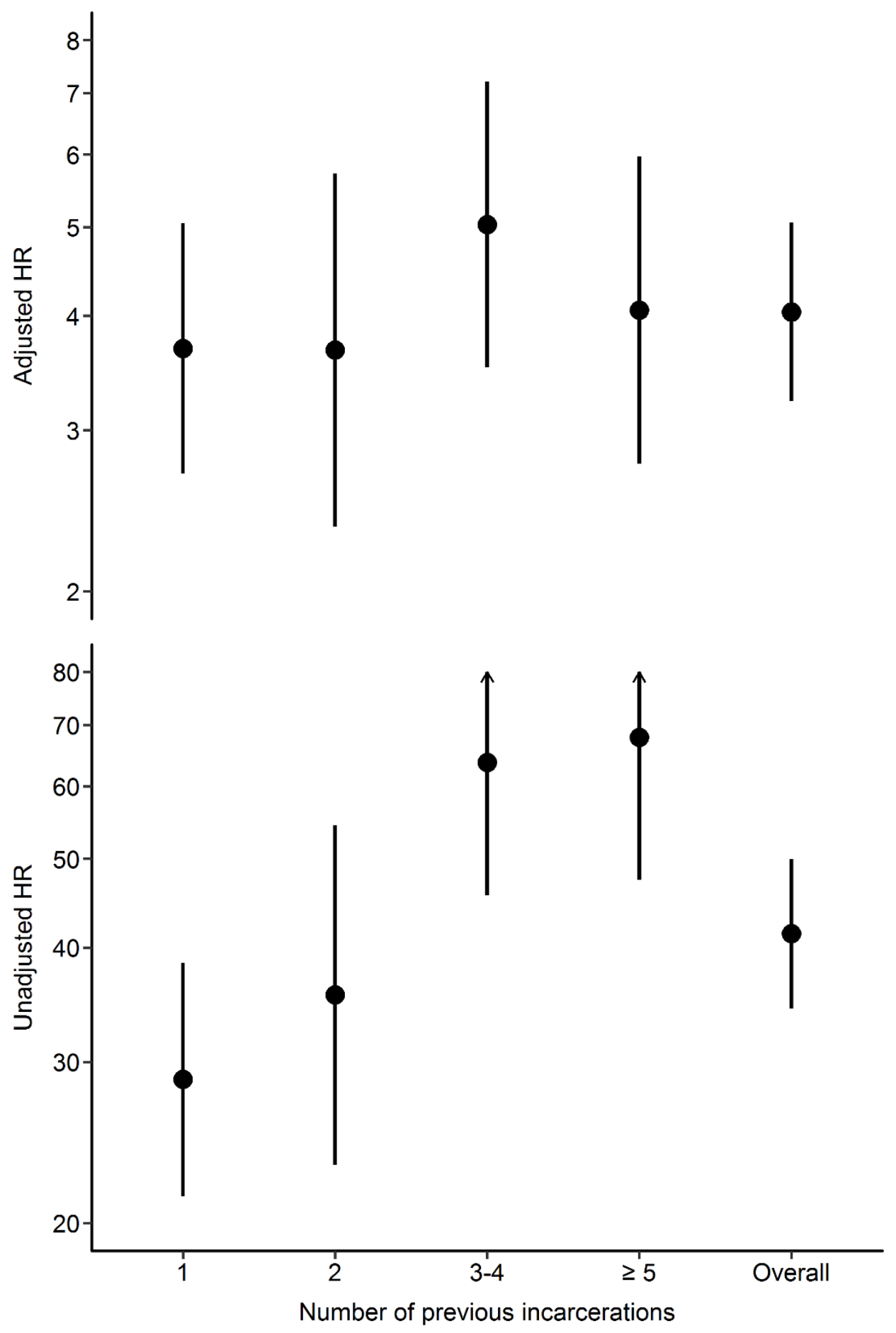

This article is protected by copyright. All rights reserved. 
Figure 2. Hazard ratios of overdose-related death for persons with an incarceration history compared with those without an incarceration history, stratified by quartiles of cumulative duration of previous incarcerations during the exposure period.

Adjusted HRs were controlled for all the characteristics in Table 1.

$\mathrm{HR}=$ hazard ratio.

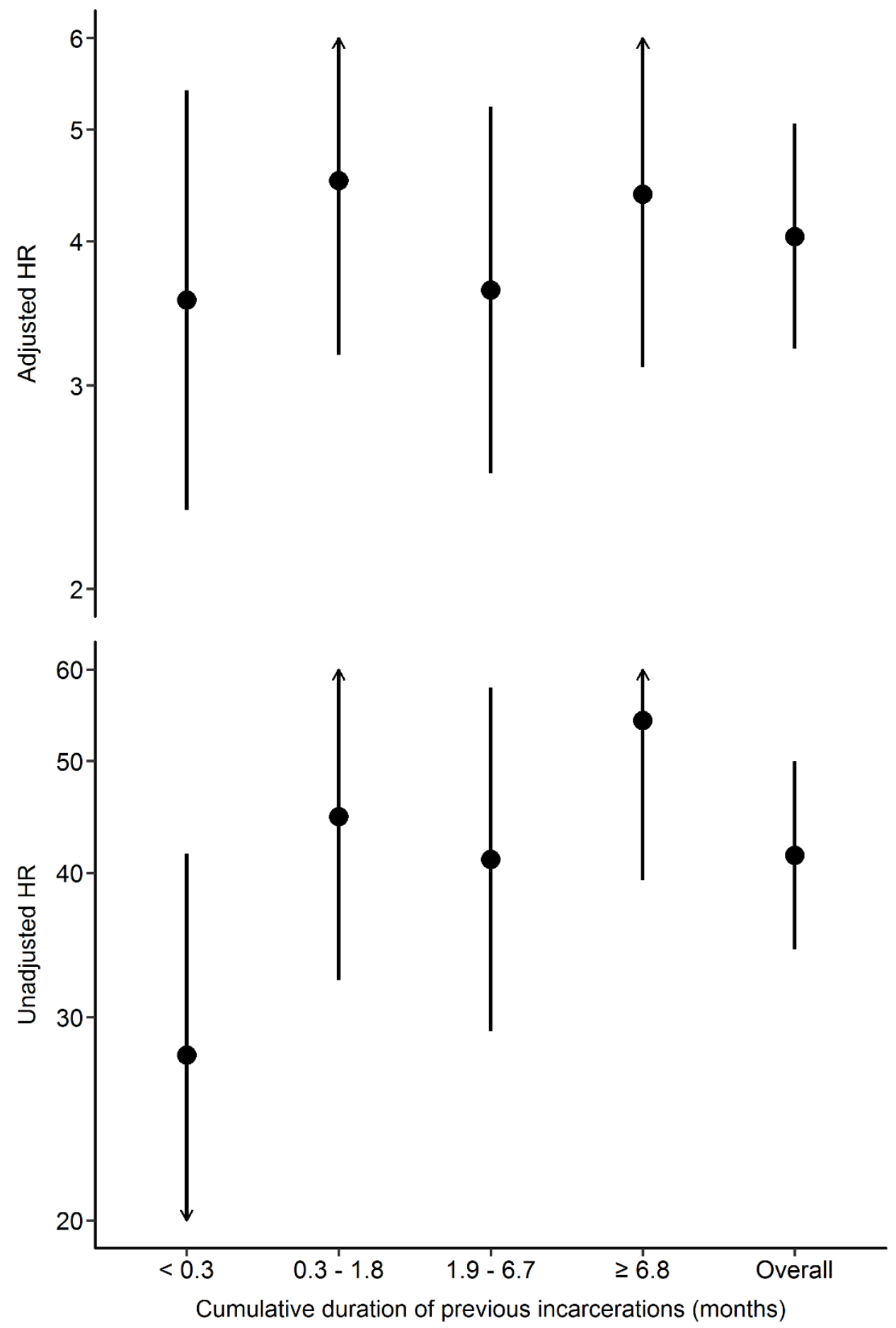

This article is protected by copyright. All rights reserved. 
Figure 3. Adjusted hazard ratios of overdose-related death for persons with an incarceration history compared with those without an incarceration history, stratified by individual and neighborhood characteristics.

Hazard ratios were adjusted for the characteristics in Table 1 without including any interaction term. $P<0.001$ for the interaction term with sex, SUD and mental illness, dispensation of benzodiazepines, and dispensation of opioids for pain; $P=0.033$ for the interaction term with NMDI.

NMDI = neighbourhood material deprivation index; SUD = substance use disorder.

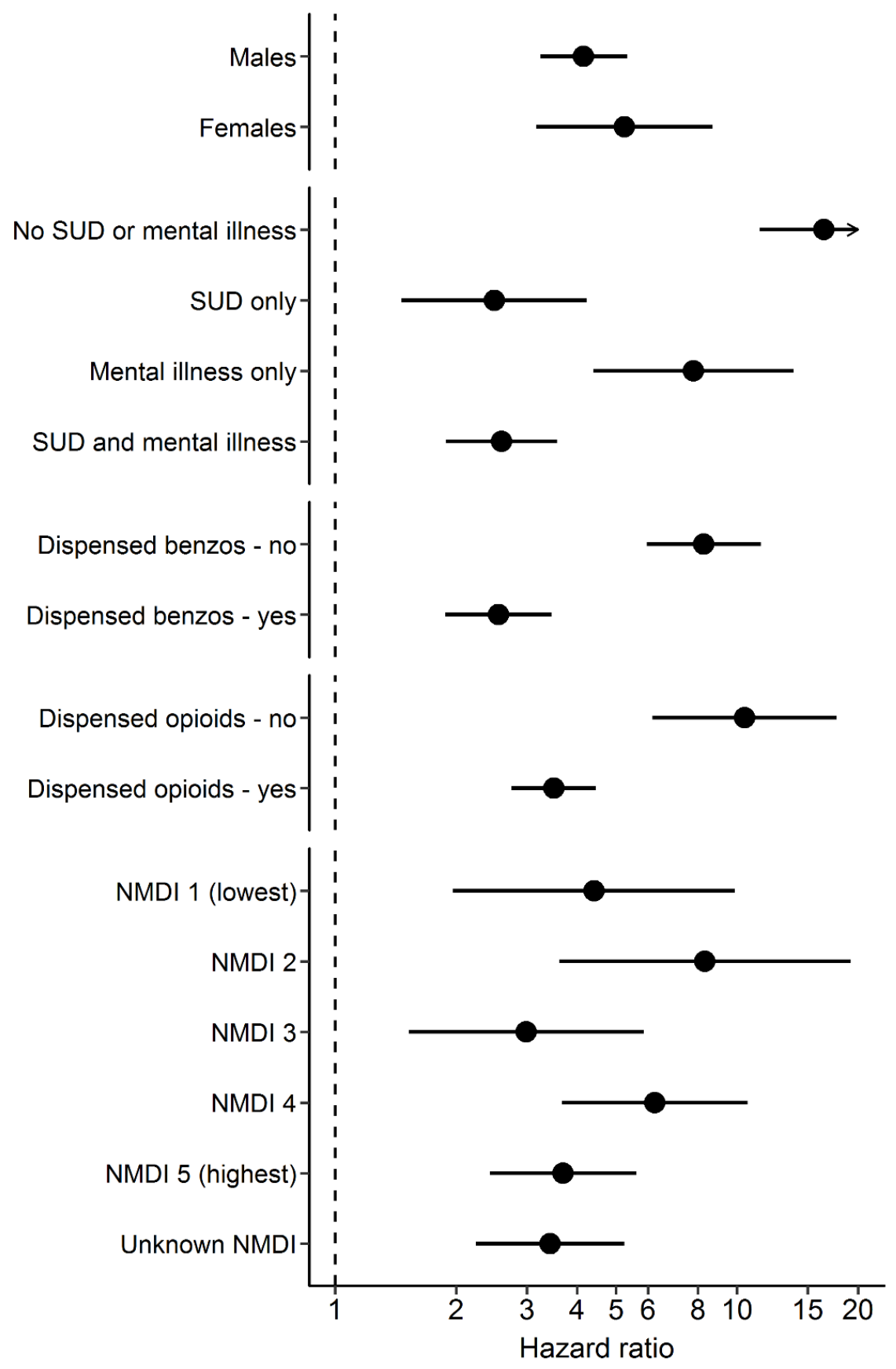




\section{REFERENCES}

1. Kouyoumdjian F. G., Kiefer L., Wobeser W., Gonzalez A., Hwang S. W. Mortality over 12 years of follow-up in people admitted to provincial custody in Ontario: a retrospective cohort study, CMAJ Open 2016; 4: E153-61.

2. Binswanger I. A., Stern M. F., Deyo R. A., Heagerty P. J., Cheadle A., Elmore J. G. et al. Release from prison--a high risk of death for former inmates, $N$ Engl J Med 2007; 356: $157-65$.

3. Binswanger I. A., Blatchford P. J., Mueller S. R., Stern M. F. Mortality after prison release: opioid overdose and other causes of death, risk factors, and time trends from 1999 to 2009, Ann Intern Med 2013; 159: 592-600.

4. Lim S., Seligson A. L., Parvez F. M., Luther C. W., Mavinkurve M. P., Binswanger I. A. et al. Risks of drug-related death, suicide, and homicide during the immediate post-release period among people released from New York City jails, 2001-2005, Am J Epidemiol 2012; 175: 519-26.

5. Spaulding A. C., Seals R. M., McCallum V. A., Perez S. D., Brzozowski A. K., Steenland N. K. Prisoner survival inside and outside of the institution: implications for health-care planning, Am J Epidemiol 2011; 173: 479-87.

6. Farrell M., Marsden J. Acute risk of drug-related death among newly released prisoners in England and Wales, Addiction 2008; 103: 251-5.

7. Ranapurwala S. I., Shanahan M. E., Alexandridis A. A., Proescholdbell S. K., Naumann R. B., Edwards D., Jr. et al. Opioid Overdose Mortality Among Former North Carolina Inmates: 2000-2015, Am J Public Health 2018; 108: 1207-13.

This article is protected by copyright. All rights reserved. 
8. Kariminia A., Butler T., Corben S., Levy M., Grant L., Kaldor J. et al. Extreme causespecific mortality in a cohort of adult prisoners--1988 to 2002: a data-linkage study, Int J Epidemiol 2007; 36: 310-6.

9. Chang Z., Lichtenstein P., Larsson H., Fazel S. Substance use disorders, psychiatric disorders, and mortality after release from prison: a nationwide longitudinal cohort study, Lancet Psychiatry 2015; 2: 422-30.

10. Graham L., Fischbacher C. M., Stockton D., Fraser A., Fleming M., Greig K. Understanding extreme mortality among prisoners: a national cohort study in Scotland using data linkage, Eur J Public Health 2015; 25: 879-85.

11. Kinner S. A., Forsyth S., Williams G. Systematic review of record linkage studies of mortality in ex-prisoners: why (good) methods matter, Addiction 2013; 108: 38-49.

12. Zlodre J., Fazel S. All-cause and external mortality in released prisoners: systematic review and meta-analysis, Am J Public Health 2012; 102: e67-75.

13. Wakefield J. Ecologic studies revisited, Annu Rev Public Health 2008; 29: 75-90.

14. Belzak L., Halverson J. The opioid crisis in Canada: a national perspective, Health Promot Chronic Dis Prev Can 2018; 38: 224-33.

15. Ministry of Mental Health and Additions. Escalating BC's response to the overdose emergency. Available at: https://www2.gov.bc.ca/assets/gov/overdoseawareness/mmha_escalating_bcs_response_report_final_26feb.pdf. (Accessed on June 26, 2020).

16. MacDougall L., Smolina K., Otterstatter M., Zhao B., Chong M., Godfrey D. et al. Development and characteristics of the Provincial Overdose Cohort in British Columbia, Canada, PLoS One 2019; 14: e0210129. 
17. BC Coroners Service death review panel: a review of illicit drug overdoses. Available at: https://www2.gov.bc.ca/assets/gov/birth-adoption-death-marriage-anddivorce/deaths/coroners-service/death-reviewpanel/bccs_illicit_drug_overdose_drp_report.pdf. (Accessed on June 26, 2020).

18. Ministry of Health. Medical Services Plan (MSP) for British Columbia (B.C.) Residents. Available at: https://www2.gov.bc.ca/gov/content/health/health-drug-coverage/msp/bcresidents. (Accessed on June 26, 2020).

19. BC Coroners Service. Illicit drug toxicity deaths in BC January 1, 2009 - December 31, 2019. Available at: https://www2.gov.bc.ca/assets/gov/birth-adoption-death-marriage-anddivorce/deaths/coroners-service/statistical/illicit-drug.pdf. (Accessed on June 26, 2020).

20. Ministry of Public Safety and the Solicitor General. A Profile of BC Corrections. Available at: https://www2.gov.bc.ca/assets/gov/law-crime-and-justice/criminaljustice/corrections/reports-publications/bc-corrections-profile.pdf. (Accessed on June 26, 2020).

21. Ministry of Health. BC Chronic Disease and Selected Procedure Case Definitions. Available at: https://www2.gov.bc.ca/assets/gov/health/conducting-health-research/dataaccess/chronic-disease-registries-case-definitions.pdf. (Accessed on June 26, 2020).

22. Menendez M. E., Neuhaus V., van Dijk C. N., Ring D. The Elixhauser comorbidity method outperforms the Charlson index in predicting inpatient death after orthopaedic surgery, Clin Orthop Relat Res 2014; 472: 2878-86.

23. Pampalon R., Hamel D., Gamache P., Philibert M. D., Raymond G., Simpson A. An areabased material and social deprivation index for public health in Quebec and Canada, Can J Public Health 2012; 103: S17-22. 
24. Therneau T. A Package for Survival Analysis in S. Available at: https://CRAN.Rproject.org/package=survival. (Accessed on June 26, 2020).

25. Schwartz R. P., Gryczynski J., O'Grady K. E., Sharfstein J. M., Warren G., Olsen Y. et al. Opioid agonist treatments and heroin overdose deaths in Baltimore, Maryland, 1995-2009, Am J Public Health 2013; 103: 917-22.

26. Pearce L. A., Min J. E., Piske M., Zhou H., Homayra F., Slaunwhite A. et al. Opioid agonist treatment and risk of mortality during opioid overdose public health emergency: population based retrospective cohort study, BMJ 2020; 368: $\mathrm{m} 772$.

27. Irvine M. A., Buxton J. A., Otterstatter M., Balshaw R., Gustafson R., Tyndall M. et al. Distribution of take-home opioid antagonist kits during a synthetic opioid epidemic in British Columbia, Canada: a modelling study, Lancet Public Health 2018; 3: e218-e25.

28. Marshall B. D., Milloy M. J., Wood E., Montaner J. S., Kerr T. Reduction in overdose mortality after the opening of North America's first medically supervised safer injecting facility: a retrospective population-based study, Lancet 2011; 377: 1429-37.

29. White J. M., Irvine R. J. Mechanisms of fatal opioid overdose, Addiction 1999; 94: 961-72.

30. World Health Organization. Preventing overdose deaths in the criminal-justice system. Available at: http://www.euro.who.int/_data/assets/pdf_file/0020/114914/Preventingoverdose-deaths-in-the-criminal-justice-system.pdf. (Accessed on June 26, 2020).

31. Dominguez-Berjon F., Borrell C., Rodriguez-Sanz M., Pastor V. The usefulness of areabased socioeconomic measures to monitor social inequalities in health in Southern Europe, Eur J Public Health 2006; 16: 54-61.

32. Krieger N. Overcoming the absence of socioeconomic data in medical records: validation and application of a census-based methodology, Am J Public Health 1992; 82: 703-10. 
33. Oquendo M. A., Volkow N. D. Suicide: A Silent Contributor to Opioid-Overdose Deaths, N Engl J Med 2018; 378: 1567-9.

34. Olfson M., Rossen L. M., Wall M. M., Houry D., Blanco C. Trends in Intentional and Unintentional Opioid Overdose Deaths in the United States, 2000-2017, JAMA 2019; 322: 2340-2.

35. BC Coroners Service. Death Investigation. Available at: https://www2.gov.bc.ca/gov/content/safety/public-safety/death-investigation. (Accessed on June 26, 2020).

36. United Nations Office on Drugs and Crime. Prevention of spread of HIV amongst vulnerable groups in South Asia. Available at: https://www.unodc.org/pdf/india/our_work_sa_prisons.pdf. (Accessed on June 26, 2020).

37. Joudrey P. J., Khan M. R., Wang E. A., Scheidell J. D., Edelman E. J., McInnes D. K. et al. A conceptual model for understanding post-release opioid-related overdose risk, Addict Sci Clin Pract 2019; 14: 17.

38. Sordo L., Barrio G., Bravo M. J., Indave B. I., Degenhardt L., Wiessing L. et al. Mortality risk during and after opioid substitution treatment: systematic review and meta-analysis of cohort studies, BMJ 2017; 357: j1550.

39. Ternes M., Goodwin S., Hyland K. Substance use disorders in correctional populations. In: M. Ternes, P. R. Magaletta, M. W. Patry, editors. The practice of correctional psychology. Switzerland: Springer Nature Switzerland AG; 2018, pp. 39-70.

40. Nosrati E., Kang-Brown J., Ash M., McKee M., Marmot M., King L. P. Economic decline, incarceration, and mortality from drug use disorders in the USA between 1983 and 2014: an observational analysis, Lancet Public Health 2019; 4: e326-e33. 
41. Dumont D. M., Brockmann B., Dickman S., Alexander N., Rich J. D. Public health and the epidemic of incarceration, Annu Rev Public Health 2012; 33: 325-39.

42. Wildeman C., Wang E. A. Mass incarceration, public health, and widening inequality in the USA, Lancet 2017; 389: 1464-74.

43. Nicholls T. L., Butler A., Kendrick-Koch L., Brink J., Jones R., Simpson A. I. F. Assessing and treating offenders with mental illness. In: M. Ternes, P. R. Magaletta, M. W. Patry, editors. The practice of correctional psychology. Switzerland: Springer Nature Switzerland AG; 2018, pp. 9-37.

44. Substance Abuse and Mental Health Services Administration. Guidelines for successful transition of people with mental or substance use disorders from jail and prison: implementation guide. Available at: https://store.samhsa.gov/product/Guidelines-forSuccessful-Transition-of-People-with-Mental-or-Substance-Use-Disorders-from-Jail-andPrison-Implementation-Guide/SMA16-4998. (Accessed on June 26, 2020).

45. Binswanger I. A., Nowels C., Corsi K. F., Glanz J., Long J., Booth R. E. et al. Return to drug use and overdose after release from prison: a qualitative study of risk and protective factors, Addict Sci Clin Pract 2012; 7: 3.

46. Western B., Braga A. A., Davis J., Sirois C. Stress and Hardship after Prison, AJS 2015; 120: $1512-47$.

47. United Nations Office on Drugs and Crime. Handbook on strategies to reduce overcrowding in prisons. Available at: https://www.unodc.org/documents/justice-andprison-reform/Overcrowding_in_prisons_Ebook.pdf. (Accessed on June 26, 2020). 


\section{University Library}

\section{- M I N E R VA}

\section{A gateway to Melbourne's research publications}

Minerva Access is the Institutional Repository of The University of Melbourne

\section{Author/s:}

Gan, WQ;Kinner, SA;Nicholls, TL;Xavier, CG;Urbanoski, K;Greiner, L;Buxton, JA;Martin, RE;McLeod, KE;Samji, H;Nolan, S;Meilleur, L;Desai, R;Sabeti, S;Slaunwhite, AK

Title:

Risk of overdose-related death for people with a history of incarceration

Date:

2020-11-27

\section{Citation:}

Gan, W. Q., Kinner, S. A., Nicholls, T. L., Xavier, C. G., Urbanoski, K., Greiner, L., Buxton, J. A., Martin, R. E., McLeod, K. E., Samji, H., Nolan, S., Meilleur, L., Desai, R., Sabeti, S. \& Slaunwhite, A. K. (2020). Risk of overdose-related death for people with a history of incarceration. ADDICTION, 116 (6), pp.1460-1471. https://doi.org/10.1111/add.15293.

Persistent Link:

http://hdl.handle.net/11343/286997 